\title{
Netzwerke knüpfen als Dienstleistung
}

\section{Sozialraumorientierung als Leitbegriff für kommunales Handeln}

Andreas Kuhn-Friedrich

In den Kommunen haben sich unterschiedliche Funktionen, Milieus und Lebensstile entwickelt, die sich räumlich konzentrieren. Das bedeutet, dass Bewohner auf unterschiedliche Weise in das Gemeinwesen integriert sind oder Schwierigkeiten damit haben, sich in das Gemeinwesen $z u$ integrieren oder akzeptiert zu werden. Damit Kommunen die unterschiedlichen Lagen, Ressourcen und Potenzialen der Bewohnerinnen und Bewohner beachten, diese nutzen oder positiv darauf einwirken können, schlägt der Deutsche Verein für öffentliche und private Fürsorge vor, dass Kommunen ihr gesamtes bewohntes Territorium nach Sozialräumen unterscheiden.

Kommunale Politik und Verwaltung tragen auf zwei Weisen dazu bei, dass »das Soziale « in Städten und Gemeinden funktioniert:

- Sie erstens schaffen und erhalten eine Infrastruktur, die ein Leben in Wohlstand ermöglicht sowie die Sozialisation der Bewohner und Bewohnerinnen $\mathrm{zu}$ Bürgern und Bürgerinnen unterstützt. Teil des Sozialen sind Sicherheit für die Bewohner und Bewohnerinnen, bezahlbarer Wohnraum in akzeptierter Qualität, eine verlässliche Versorgung und Entsorgung. Wer diese Leistungen wie erbringt, ist zwar Gegenstand von Entscheidungen geworden, aber diese Entscheidungsdisposition ändert nichts an der Gewährleistungsverantwortung

Dr. Andreas Kubn-Friedrich ist wissenschaftlicher Referent im Deutschen Verein für öffentliche und private Fürsorge und dort im Arbeitsfeld »Planung, Steuerung und Qualifizierung der sozialen Arbeit und der sozialen Dienste « zuständig für die

Themenbereiche Qualitätsmanagement, Evaluation, bürgerschaftliches

Engagement.

E-Mail

kuhn-friedrich@deutscher-verein.de kommunaler Politik und Verwaltung.

- Kommunale Politik und Verwaltung haben zweitens die Aufgabe, gesetzlich und rechtlich geregelte Ansprüche einzelner Personen auf Hilfe und Unterstützung oder Schutz einzulösen (bei Hilfe für Menschen mit Behinderung gilt das - bisher - für die Mehrheit der Bundesländer, nicht für alle). Dafür ist ein ausdifferenziertes System von Behörden, Ämtern und Dienstleistungen geschaffen worden. Wenn freie und gewerbliche Anbieter diese Leistungen erbringen, ändert auch das nichts daran, dass kommunale Politik und Verwaltung dafür verantwortlich sind, dass dies in dem notwendigen Maß, in geeigneter Weise und wirtschaftlich geschieht. Die Verantwortlichen nennen das Pflichtaufgaben.

Im Vergleich dazu ist sozialräumliches Handeln für kommunale Politik und Verwaltung neu. Da sie Pflichtaufgaben als Einzelhilfe verstehen, ist sozialräumliches Handeln eine freiwillige Aufgabe, so etwas wie ein Luxus, den sich einige Kommunen leisten und andere nicht. Mit diesem Verständnis kommunaler Sozialpolitik fällt es den meisten Akteuren in Politik und Verwaltung schwer, ihre Kommune als »Sozialraum « zu verstehen und daraus praktische Konsequenzen zu ziehen. Auch Stadtplanung und Stadtentwicklung wurden lange Zeit als Entwicklung von Infrastruktur, als Hoch- und Tiefbau begriffen und betrieben. In vielen Kommunen ist das auch heute noch so. Die Infrastrukturförderung und Einzelhilfe erzielte lange Zeit die gewünschten "sozialen Effekte", solange und in dem Maße, wie die Bewohnerinnen und Bewohner sich durch Familien, Nachbarschaften, Bildungseinrichtungen, Kirchen und Erwerbsarbeit zu Bürgern und Bürgerinnen sozialisierten, auf die kommunale Politik und Verwaltung "zählen « und sich verlassen können, die einen gemeinsamen oder sozial zuordenbaren Kanon von Normen, Werten und Verhal- tensweisen zu ihrem eigenen gemacht haben, die immer auch bereit und in der Lage sind, sich ehrenamtlich oder sozial zu engagieren, wenn das erforderlich ist oder das ohnehin tun.

Diese Sozialisation findet immer weniger statt und die genannten Institutionen der Integration funktionieren immer weniger. Sozialisation ist heute Individualisierung, und Integration findet heute für viele Menschen in Milieus statt, über Quartiere und Nachbarschaften (nicht über klassische Bildung und Erwerbsarbeit). In Stadt- und Ortsteilen vieler Kommunen sind Sozial- und Jugendhilfe für einen großen Teil der Bewohnerinnen und Bewohner eine Lebensgrundlage und für die zuständigen Behörden und Dienstleister ein Massengeschäft geworden.

Hilfebedarfe sind räumlich konzentriert und gerade in solchen Räumen haben Quartiere eine zunehmende Bedeutung für die Bewohner und Bewohnerinnen. Deshalb ist der geografische Raum, das Quartier, zwar nicht deckungsgleich mit individuellen Sozialräumen, aber gerade in sogenannten benachteiligten Gebieten ein wesentlicher Faktor individueller Sozialräume.

Diese Diagnose ist ein Ausgangspunkt für »Eckpunkte sozialräumlicher Ausgestaltung kommunalen Handelns «, die der Deutsche Verein im Juni 2008 beschlossen hat (vgl. Nachrichtendienst des Deutschen Vereins, August 2008, 377-384).

Die Perspektive der Eckpunkte ist jedoch nicht auf benachteiligte Gebiete ausgerichtet, sondern auf das gesamte Gebiet einer Kommune. Die Eckpunkte empfehlen, das gesamte Gebiet einer Kommune als Sozialräume zu betrachten. Sie postulieren, dass das mit vorhandenen Statistiken und ergänzenden Erhebungen vor Ort möglich ist. Wie Sozialraumanalysen in Kommunen so zu machen sind, dass daraus Handlungskonsequenzen und Prioritäten abgeleitet werden können, wird Gegenstand weiterer Empfehlungen sein. 
Die Eckpunkte des Deutschen Vereins betonen, dass Politik und Verwaltung sozialräumliches Handeln auf den Weg bringen und unterstützen müssen, wenn dieses Handelns erfolgreich sein soll. Politik und Verwaltung müssen dazu personelle und finanzielle Ressourcen bereitstellen. Planung und Maßnahmen in Stadt- und Ortsteilen beteiligen die Bewohner und Bewohnerinnen nicht nur,
Bewohner nach ihren Möglichkeiten sozial integriert und möglichst selbstständig leben und ein friedliches Miteinander (oder mindestens Nebeneinander) möglich ist.

Eine Schwierigkeit, die sozialräumliches Handeln auszeichnet und auch immer wieder scheitern lässt, ist die Kommunikation zwischen Politik, Verwaltung, lokalen Akteuren und Bewohnerin-

\section{"Was sozialräumliches Handeln immer wieder scheitern lässt, ist fehlende Kommunikation zwischen den Beteiligten «}

sondern arbeiten mit ihnen zusammen und setzen auf deren Engagement. Die Ansprache vor Ort und ein »Raum « für Eigeninitiative und eigene Gestaltung sind bedeutsame Faktoren dafür, dass die Ressourcen und Potenziale der Bewohnerinnen und Bewohner aktiviert und genutzt werden können, um Quartiere zu entwickeln. Quartiere sollen so entwickelt sein, dass die Bewohnerinnen und nen und Bewohnern. Für Befürworter einer bürgerschaftlichen Stadtentwicklung sind $»$ kommunale Institutionen potenziell engagementfeindlich «. Aber es ist meistens nicht böser Wille, wenn kommunale Verwaltung die Bewohnerinnen und Bewohner nicht versteht oder deren Anliegen nicht eins zu eins aufnimmt, sondern eine andere Perspektive und ein anderer Code, in dem kommuniziert wird.

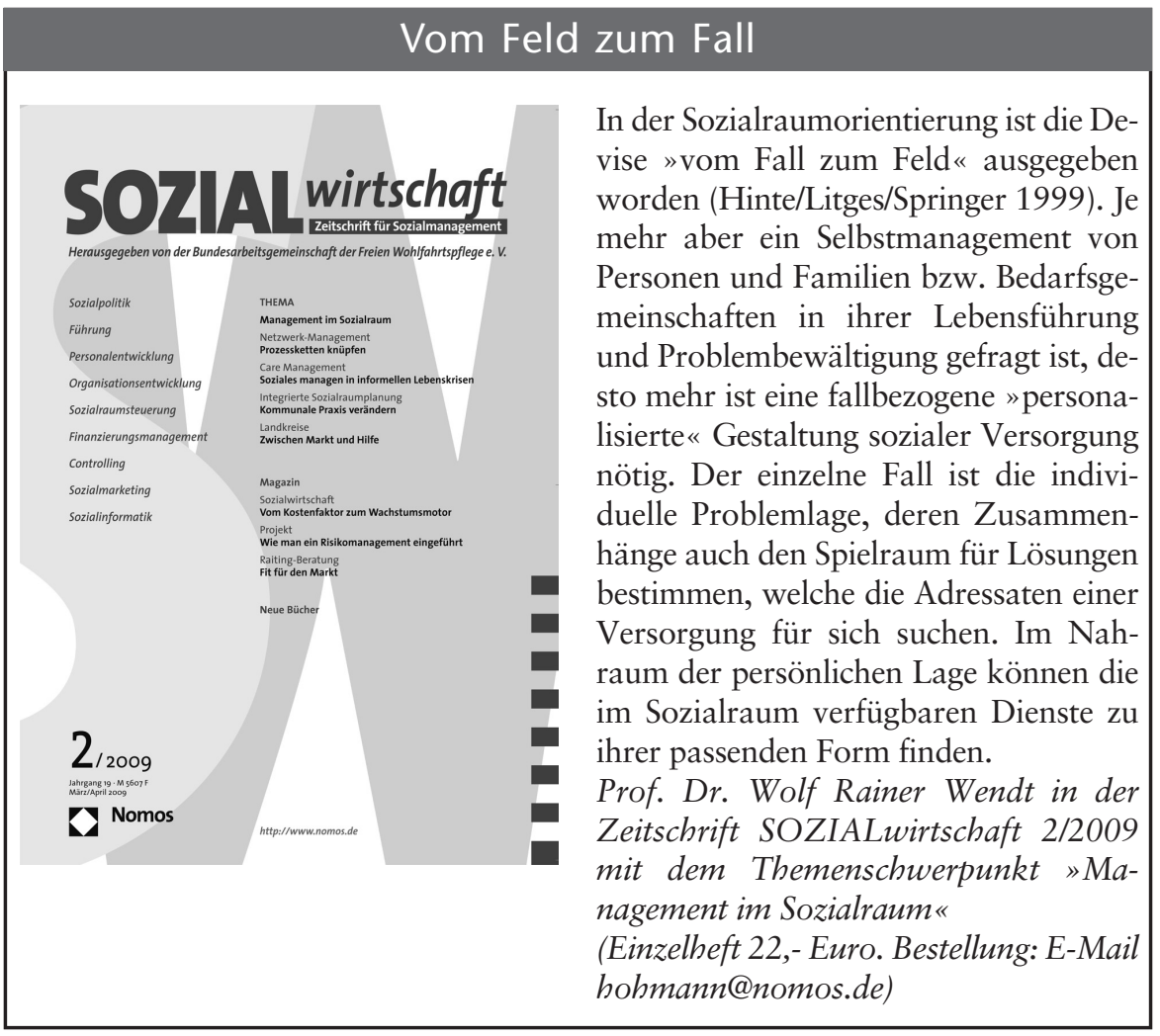

In den Eckpunkten zum sozialräumlichen Handeln stellt der Deutsche Vereins fest, dass besondere Arrangements notwendig sind, damit Politik und Verwaltung, lokale Akteure sowie Bürgerinnen und Bürgern so miteinander kommunizieren können, dass sozialräumliche Entwicklung möglich ist. Dazu werden intermediäre Organisationen, Organisationsformen und Instanzen gebraucht. Es ist eine offene Frage, ob Quartiersmanager und Quartiersmanagerinnen mit Quartiersbeiräten das sein können oder sogenannte »Lokale Entwicklungspartnerschaften «. Oder ob es »Bürgerplattformen « des Community Organizing geben muss, damit Bewohnerinnen und Bewohner bei Politik und Verwaltung mit Ihren Anliegen Gehör und Unterstützung finden.

\section{Wie sozialräumliches Handeln gelingt}

In jedem Fall wird für sozialräumliches Handeln eine besondere Dienstleistung gebraucht: Netzwerker und Netzwerkerinnen, die auf vielfältige Weise Akteure vor Ort sowie Bewohnerinnen und Bewohner zusammenbringen. Wenn das auf Dauer und kontinuierlich gelingt, ist das schon ein Teil der Lösung vieler Probleme in Quartieren. Vorschläge für die Lösung akuter Probleme finden sich dann fast von selbst und sie umzusetzen ist dann keine Last, sondern eine Bereicherung für alle Beteiligten.

Solche »Dienstleister « können Engagement und Kooperation nicht produzieren, aber sie können Engagement anregen und produktiv machen und Gemeinsinn stiften. Die Rolle des Geldes bei der sozialen Entwicklung von Stadt- und Ortsteilen wird in den Eckpunkten des Deutschen Vereins nicht ausgearbeitet, sehr wohl aber skizziert und an Beispielen erläutert. 